\title{
Distant heterotopic callosal connections to premotor cortex in non-human primates
}

Lanz F. ${ }^{1}$, Moret V. ${ }^{1}$, Ambett R. ${ }^{1}$, Cappe C. ${ }^{2}$, Rouiller E.M. ${ }^{1}$, Loquet G. ${ }^{1 *}$

${ }^{1}$ Department of Medicine, Domain of Physiology and Fribourg Cognition Center, University of Fribourg, Chemin du Musée 5, CH-1700 Fribourg, Switzerland

${ }^{2}$ Centre de recherche Cerveau et Cognition (CerCo), CNRS, Université de Toulouse - UPS, 31052 Toulouse, France

* Corresponding author : gerard.loquet@unifr.ch; Phone.: +41 2630087 69; Fax: +41 26300 9734

\begin{abstract}
Cortico-cortical connectivity has become a major focus of neuroscience in the last decade but most of the connectivity studies focused on intrahemispheric circuits. Little has been reported about information acquired and processed in the premotor cortex and its functional connection with its homotopic counterpart in the opposite hemisphere via the corpus callosum. In nonhuman primates (macaques) lateralization is not well documented and its exact role is still unknown. The present study confirms in two macaques the existence of homotopic contralateral projections and completes the picture by further exploring heterotopic (nonmotor) callosal projections. This was tested by injecting retrograde tracers in the premotor cortical areas PMv and PMd (targets). Our method consisted of identifying the connections with all the homo- and heterotopic cortical areas located in the contralateral hemisphere. The results showed that PMd and PMv receive multiple low density labelled inputs from the opposite heterotopic prefrontal, parietal, motor, insular and temporal regions. Such unexpected collection of transcallosal inputs from heterotopic areas suggests that the premotor areas communicate with other modalities through long distance low density networks which could have important implications in the understanding of sensorimotor and multimodal integration.
\end{abstract}




\section{Keywords}

neuroanatomy; cortical connectivity; corpus callosum; premotor cortex; non-human primate; multisensory integration

\section{Author contributions}

Conceived and designed the experiment: EMR, CC. Performed the experiment: EMR, CC. Analyzed the data: FL, VM, EMR, GL. Wrote the paper: GL, RA, EMR, FL.

\section{$\underline{\text { Introduction }}$}

In placental mammals the corpus callosum is the main commissural structure (versus the anterior commissure) which connects homotopic and heterotopic regions of the cerebral cortex between the two hemispheres (e.g. Innocenti 1986; 1994; 1995; Aboitiz et al. 2003). The identification of the topography of these connections is still in progress in humans especially through functional magnetic resonance imaging (Fabri et al. 2011; Phillips and Hopkins 2012) and diffusion tensor imaging (Hofer and Frahm 2006; Phillips and Hopkins 2012). In non-human primates, the majority of brain connectivity data (see datasets established based on the work of Paxinos et al., 2000; Van Essen, 2002; Dubach and Bowden, 2009; Rohlfing et al., 2012; Markov et al., 2014; Calebrese et al., 2015) originate from one hemisphere based on the assumption (though unproven) that lateralization does not play a key role in macaques'. The few available studies (e.g. Pandya and Vignolo, 1971) state that callosal connections predominantly link homotopic cortical regions. This view has been questioned in the past decade (Clarke 2003) and new evidence of numerous and widespread heterotopic callosal connections have emerged in human studies. For example, in the visual cortex, heterotopic connections to the opposite hemisphere have been identified (Clarke and Miklossy 1990; Clarke 1994). Visual connections have also been reported from the inferior temporal cortex (associated with visual recognition) to the contralateral temporoparietal junction referred to as Wernicke's area in humans (associated with speech comprehension) and to the inferior frontal gyrus (Broca's area associated with speech production) (Di Virgilio and Clarke 1997). In the motor cortex, corticocortical connectivity between motor areas and the other hemisphere has been identified in non-human primates (e.g. Pandya and Vignolo, 1971; Jenny 1979; Rouiller et al. 1994; Liu et al. 2002; Marconi et al. 2003). In 2005, Boussaoud et al. showed that the premotor cortical areas PMd-c (F2) and PMd-r (F7) receive 
heterotopic inputs from contralateral pre-SMA (F6) and that PMd-r was strongly connected with prefrontal cortex. According to those authors callosal afferent connectivity to PMv-c (F4) was broader than that to PMv-r (F5). Other authors (Marconi et al. 2003) reported that the major heterotopic callosal projection to F7 originated from F2 followed by weaker inputs from pre-SMA (F6), area 8 (FEF) and prefrontal cortex (area 46). The same authors showed that the heterotopic inputs to F2 mainly emanated from F7 followed by a smaller contingent coming from F5, F4, SMA-proper (F3) and F1. These reports suggest that premotor areas connectivity is composed of sets of heterotopic inputs originating from a mosaic of motor areas. Furthermore, in the premotor cortex, touch, vision and/or hearing inputs have been found (Weinrich and Wise 1982; Weinrich et al. 1984; Graziano et al. 1997; Graziano et al., 1999) contributing to sensorimotor transformation (Blanchard et al., 2013). Those heterotopic or multisensory inputs would gain in being further studied since they provide a basis for underlying voluntary actions directed to a goal, more efficiently when more than one sensory modality is engaged (Stein and Meredith 1993; Giard and Peronnet 1999; Driver and Noesselt 2008).

Most available tracing studies in monkeys (see above) on callosal motor connectivity were based on injections restricted to a specific cortical subarea (e.g. F2, F3, F4 or F5 in PM; F3 or F6 in SMA) or even to a limited body part (hand area in F1 and F3). In order to establish a more comprehensive callosal connectivity pattern, the present study is based on larger tracer injections covering a large part of the dorsal premotor cortex (PMd) and the ventral premotor cortex (PMv), respectively. We tested the hypothesis that both PMd and PMv receive significant direct heterotopic non-motor callosal inputs from the prefrontal, parietal and temporal lobes, which may amount up to $5 \%$ of the total callosal projections in each of these lobes.

\section{$\underline{\text { Experimental Procedures }}$}

Two non-human primates (Mk-CI Macaca mulatta and Mk-R9 Macaca fascicularis), 3 and 4 years old and weighing 3 and $4 \mathrm{kgs}$, respectively, were re-used from a previous study on thalamocortical and corticothalamic projections (Cappe et al. 2007; 2009). The study was conducted according to both the guidelines of the National Institute of Health (Guide for the Care and Use of Laboratory Animals, NIH Publication $N^{\circ} 80-23$, revised in 1996), those of the European Community (Guidelines for Animals Protection and Use for Experimentation, $86 / 609 / E E C$ ), and approved by local (Swiss) veterinary authorities (authorization $\mathrm{N}^{\circ} 156 / 04$ 
and 156/02). All efforts were made to minimize the number of animals used and their suffering. The present work is based on the same injections of 4 neuroanatomical retrograde tracers (see Table 1) as described in a previous report (Cappe et al. 2009) but considered here for the callosal connectivity. Briefly, the animals were pre-medicated with ketamine ( 5 $\mathrm{mg} / \mathrm{kg}$, i.m.), Carprofen as an analgesic (Rymadil, $4 \mathrm{mg} / \mathrm{kg}$, s.c.), antibiotics (Albipen, ampicillin $10 \%, 15-30 \mathrm{mg} / \mathrm{kg}$ diluted $1: 1$ in saline, i.m. $)$, atropine sulfate $(0.05 \mathrm{mg} / \mathrm{kg}$, i.m. $)$ and dexamethasone (Decadron, 0.02-0.3 mg/kg/day diluted 1:1 in saline, i.m.). Then, the monkeys were anesthetized with propofol $(0.1-0.3 \mathrm{mg} / \mathrm{kg} / \mathrm{min}$, i.v.) and placed in a stereotaxic frame under aseptic conditions. The skull and the dura mater on the left side were opened over the premotor cortex. In the frontal lobe, PMd and PMv were localized based on the position of the central and arcuate sulci and the boundary between both areas was established based on the genu of the arcuate sulcus (Liu et al. 2002; Morel et al. 2005).

\begin{tabular}{|l|c|c|c|c|}
\hline Animal & $\begin{array}{c}\text { Injection } \\
\text { site }\end{array}$ & Tracer & $\begin{array}{c}\text { Volume } \\
(\mu \mathrm{l})\end{array}$ & $\begin{array}{c}\text { Number of } \\
\text { sites injected }\end{array}$ \\
\hline Mk-R9 & PMd & $\begin{array}{c}\text { Fluoroemerald (FE) } \\
\text { Molecular Probes, Eugene, OR }\end{array}$ & 10 \\
\cline { 2 - 5 } & PMv & $\begin{array}{c}\text { Fast Blue (FB) } \\
\text { Fluka, Switzerland }\end{array}$ & 3.5 & 7 \\
\hline Mk-CI & PMd & $\begin{array}{c}\text { Diamidino Yellow (DY) } \\
\text { Sigma Aldrich, France }\end{array}$ & 5.1 & 7 \\
\cline { 2 - 5 } & PMv & $\begin{array}{c}\text { Cholera toxin B subunit (CB)* } \\
\text { List Biological Laboratories, Campbell, CA }\end{array}$ & 1.9 & 72 \\
\hline
\end{tabular}

Table 1

Summary of injection sites, tracers, volumes and number of sites injected in the two macaques Mk-R9 and Mk-CI. Representations of the injection sites are available in Figures 1 and 2. *: Due to an error of transcription in Cappe et al. (2009), the tracer injected in PMv in Mk-CI is indeed CB and not WGA, as indicated by mistake in Cappe et al. (2009): in their legend of Fig.1, "WGA" should be replaced by "CB". The data are however not affected, as the Figure 1 of Cappe et al. (2009) indeed describes the data for PMv, derived from CB injection.

Injections of the tracers were executed by using 5 to $10 \mu$ l Hamilton syringes inserted perpendicularly to the cortical surface. Then, the dura mater, muscles and skin were sutured 
and the monkeys were treated for several days with an analgesic (Rymadil, $5 \mathrm{mgkg}, \mathrm{p.o.}$ ) and an antibiotic (Amoxicilline, $10 \mathrm{mg} k \mathrm{~kg}$, p.o.). Following a survival period of 2 to 3 weeks, the animals were deeply anesthetized, given a lethal dose of sodium pentobarbital (Vetanarcol 90 $\mathrm{mg} / \mathrm{kg}$ i.p.) and were perfused transcardially with first $0.3 \mathrm{~L}$ saline $(0.9 \%)$ then $3 \mathrm{~L}$ paraformaldehyde ( $4 \%$ in phosphate buffer $0.1 \mathrm{M}, \mathrm{pH}=7.4$ ), with a mixture $(2 \mathrm{~L})$ of paraformaldehyde $4 \%$ and sucrose $10 \%$ (in phosphate buffer) and finally with sucrose 20 and $30 \%$ ( $2 \mathrm{~L}$ in phosphate buffer).

The histological processing of the brain has also been described in detail in previous reports by Morel et al. (2005), Cappe et al. (2007) and Cappe et al. (2009). In summary, first the brain was sectioned in the frontal plane $(40 \mu \mathrm{m}$ sections) on a freezing microtome. The sections were collected in five series among which one was immediately mounted on slides and stored in the refrigerator for fluorescent microscopy analysis. The plotting of labelled neurons with fluorescent and/or non-fluorescent tracers was done using the MicroBrightField Neurolucida System (Colchester, USA). Drawings of cortical contours in Nissl-stained sections were imported in Neurolucida's system in order to be overlapped with the analyzed sections to identify the cortical areas. Complete drawings including the plots of labelled cells were then exported to the software CorelDrawX6 (Version 16, 2012) and cell counting was performed. The quantitative analysis was conducted by calculating for each tracer the percentage of cells labelled in one cortical area against the total number of cells labelled with this particular tracer in the whole hemisphere. Such percentage distribution as a function of a cortical area was represented first in histograms and grouped according to anatomical location. Second, the strength of the callosal connections between the multiple areas was represented in the form of a color weighted connectivity matrix as done by others (e.g. Markov et al., 2014). This matrix used a logarithmic scale which was then translated into a positive scale where values covered 3 equal ranges from 0 to 1.25 corresponding to sparse connections, from 1.25 to 2.5 corresponding to moderate connections and, greater than 2.5 corresponding to strong connections.

\section{$\underline{\text { Results }}$}

\section{Injection sites}

In the present study, 2 out of the 6 cortical areas injected in Cappe et al. (2009) were reinvestigated for callosal connectivity: the dorsal and the ventral premotor cortices (PMd and $\mathrm{PMv}$ ). Repeated injections of different retrograde tracers extended anteriorly from the genu of 
the arcuate sulcus (Figure 1, 2 and 3) to the caudal end of the spur of the arcuate sulcus. The injections sites were in F7/F2 (learning-related area [Brasted and Wise, 2004] modulated by eye movement [Boussaoud, 1985]/guiding reaching area [Cisek and Kalaska, 2005]) and in F4/F5 (sensory guidance of movement [Graziano et al., 1994] and peripersonal space [Fogassi et al., 1996]/hand shaping during grasping, vocalization [Coudè et al., 2011] and mirror neurons [Kohler et al., 2002]). Examples of the general distribution of retrogradely labeled neurons with FB, FE, DY and CB are presented in Figure 3. The relative position of each coronal section (as well as their estimated stereotaxic level in $\mathrm{mm}$ from interaural axis) is indicated on a schematic brain map based on the monkey brain atlas of Saleem and Logothetis (2007).

\section{Injections in PMd}

Figure 4, upper panel, shows the distribution of retrograde labelling in the hemisphere contralateral to the injection of tracers FE and DY in PMd. The distribution of callosal inputs to PMd with respect to their lobe of origin is indicated in Table 2. In Mk-R9 the most abundant retrograde labelling was found in motor areas (69.1\%). Less dense labelling was observed in the temporal lobe (areas TE+TPO; 9.1\%), the parietal lobe (3a/b, 1-2, SII; 9.7\%), the prefrontal cortex (8.4\%, in particular areas 44 and 45$)$ and the insular cortex (2.9\%). In Mk-CI the main labelling has been obtained in motor areas (94.3\%) followed by the prefrontal cortex $(5.7 \%$, area 9). In terms of origin of the callosal cortical projections, callosal inputs to PMd originate mainly from motor cortical areas. To a lesser extent, moderate transcallosal projections came from the temporal and the parietal lobe in one animal (Mk-R9) and from the prefrontal cortex (Mk-R9 \& Mk-CI). Sparse callosal projections were identified coming from the insula (Fig. 4).

\begin{tabular}{|l|c|c|}
\hline & Mk-R9 & Mk-CI \\
\hline Parietal & $9.7 \%$ & $0 \%$ \\
\hline Prefrontal & $8.4 \%$ & $5.7 \%$ \\
\hline Motor & $69.1 \%$ & $94.3 \%$ \\
\hline Insula & $2.9 \%$ & $0 \%$ \\
\hline Temporal & $9.1 \%$ & $0 \%$ \\
\hline
\end{tabular}

Table 2

Summary of distribution of retrogradely labelled callosal inputs to PMd. 


\section{Injections in PMv}

Figure 4, lower panel, illustrates the histogram distribution of retrograde labelled cells in the hemisphere contralateral to the injection of 2 tracers (FB and $\mathrm{CB}$ ) in PMv of the two monkeys. The distribution of callosal inputs to PMv with respect to their lobe of origin is indicated in Table 3. In Mk-R9 these injections have labelled cells mainly in motor areas (78\%): area 24, SMA-proper (F3), F2, F4 and F5. A moderate labelling was found in the prefrontal $(9.8 \%)$, parietal $(7.0 \%)$ and insular $(5.1 \%)$ regions. No retrograde labelling was observed in the temporal lobe. In Mk-CI, the main (homotopic) labelling was found in motor areas (83.4\%): F5, F4, frontal eye field area 8A, F3, F2 and in the cingulate cortex (area 24). Less dense labelling was noticed in the other lobes: prefrontal, parietal, insula and temporal lobes. Put into connectivity perspective callosal inputs to PMv mainly originated from contralateral premotor cortices. To a lesser extent, moderate to weak callosal inputs were identified from the FEF, the precentral operculum, somatosensory cortices (SII and 1-2), the area $\mathrm{G}$ (terminal plexus in gustatory cortex) and the insula. Finally, sparse callosal connections originated from a large palette of cortical areas (Fig. 4).

\begin{tabular}{|l|c|c|}
\hline & Mk-R9 & Mk-CI \\
\hline Parietal & $7.0 \%$ & $3.8 \%$ \\
\hline Prefrontal & $9.8 \%$ & $11.3 \%$ \\
\hline Motor & $78.0 \%$ & $83.4 \%$ \\
\hline Insula & $5.1 \%$ & $1.2 \%$ \\
\hline Temporal & $0 \%$ & $0.3 \%$ \\
\hline
\end{tabular}

Table 3

Summary of distribution of retrogradely labelled callosal inputs to PMv.

\section{Connectivity matrices}

In order to assess more quantitatively the respective cortical areas of origin, connectivity matrices were established. Figure 5 shows the individual connectivity matrices for Mk-R9 and Mk-CI. This matrix has been obtained based on the calculated ratio of labeled neurons with one marker in a specific cortical area relative to the total number of labeled neurons with the same marker over the hemisphere opposite to the injection site. These values have been turned into logarithms then translated to a positive scale in order to quantify connection weights. In Figure 5, each column gives the calculated connection weight for each animal per area (PMv and PMd) and each row the origin of its transcallosal inputs originating from 41 
separate cortical areas. According to the colorbar used, bright colors represented strong connections whereas dark colors represented weaker connections. The results show strong callosal homotopic connections especially at F4, F5, F2 levels. Other strong links with PMv and PMd were observed with heterotopic areas F2, F3, F4, F5 and 24. Moderate non-motor heterotopic connections are also well present in both monkeys but show less homogeneity in relation with PMd. Therefore only areas $8 \mathrm{~A}$ and $8 \mathrm{Bs}$ can be noted as moderately connected to PMd whereas PrCo, the insula, the area G, the parietal and the prefrontal lobes show moderate connections to PMv. As far as sparse heterotopic connections are concerned, the sources to PMv originate essentially from the parietal and the prefrontal lobes in both animals. In contrast, for PMd, only one animal (Mk-R9) displays sparse heterotopic connections with the parietal and the prefrontal lobes but also with the temporal lobe.

\section{$\underline{\text { Discussion }}$}

Our results are in agreement with the hypothesis that both PMd and PMv receive multiple heterotopic non-motor callosal inputs from the contralateral prefrontal, parietal and possibly the temporal lobes. Those projections are few in quantity compared with the homotopic ones but represent a non-negligible amount (up to 5 to $7 \%$ ) and therefore may be functionally relevant.

\section{Connectivity of PMd and PMv}

Across the two animals of different species, it appears that the homotopic projections (Figures 4 and 5) are relatively consistent, suggesting that injections are comparable. Those repeated injections were executed in two different cortical subregions (F2/F7 and F4/F5; see Figures 1 and 2) and revealed strong homotopic corticocortical connections with the opposite hemisphere as described earlier by others (Pandya and Vignolo, 1971; Rouiller et al. 1994; Marconi et al. 2003). In addition to these major homotopic inputs linking bilaterally the F3 areas, the cingulate motor areas (Rouiller et al. 1994), F7, F2, F4, F5 (Boussaoud et al., 2005), smaller contingent of callosal inputs to PMd or PMv originating from contralateral heterotopic areas were described with PMd connected with area 46 of the prefrontal cortex, F6, F5, F4, F3, F2, F1 and area 8 (Marconi et al., 2003). The present study reports the same patterns of homo and heterotopic callosal connections to PMd and PMv (see Figures 4 and 5) and completes it with some other non-motor contingent of callosal projections to PMv (1-2, 
3a/b, SII, 44, 45, area 12, 24, G, Insula, PrCo) identified in both animals and to PMd (area 45, 24). The present observations mean that globally PM receives more contralateral nonhomotopic projections than expected (Marconi et al., 2003; Boussaoud et al., 2005). We can add to these results the identification of sparse heterotopic connections originating from the parietal cortex (area 1-2, 3a/b, 5, 7, SII, AIP/LIP/VIP), the prefrontal cortex (44, 45, area 9, 24), the auditory cortex (R, AI, RM, CM, ML, AL, RTL) or the temporal gyrus (STG, TAa, TPO, PGa, TE, IPa) to PMd in one animal (Mk-R9). Such differences between our two animals may be due to differences in connectivity between the two species but also differences at the injection site (e.g. marker spread, precise location of the injection, layers distribution) or because of the tracer type (e.g. sensitivity, specificity, single tracing). Without bringing a definite answer to this point, one can say that our approach using injections covering a large part of the premotor cortex was appropriate since it allowed us to describe long-distance projections whose functional significance (though still unknown) is probably not related to the quantity of neurons of origin.

\section{Intrahemispheric versus callosal connections}

In the motor system, reports demonstrated that intrahemispheric connectivity is formed by a series of interconnected areas working hierarchically (Keele et al. 1990; Grafton and Hamilton 2007) and/or in parallel (Rizzolatti et al. 1998; Rizzolatti and Luppino 2001). For example the neurons projecting to F1 were shown to originate mainly from PMd, PMv, SMA according to a somatotopic organization (Ghosh et al. 1987; Godschalk et al. 1984) and from other networks like the somatosensory areas 3a, 1-2, SII, the posterior parietal cortex and the cingulate cortex (Morecraft et al., 2012). Similarly PMd and PMv were shown to be connected to many other cortical areas located in the same hemisphere in the frontal and parietal lobes and to a lesser extent in the temporal lobe (Matelli et al. 1984; Barbas and Pandya 1987; Kurata 1991; Morecraft et al., 2012). Interestingly these two premotor areas have already been reported to receive inputs from associative "sensory" areas like MIP, AIP, 7a and 7b (Matelli et al. 1986; Ghosh and Gattera, 1995; Wise et al. 1997; Tanné-Gariépy et al. 2002) known to have visual properties. Therefore these premotor areas were further investigated recently from a multisensory perspective (Lanz et al. 2013) in order to better understand their involvement in sensory-motor transformations. However, although these pathways and processes were generally found in one hemisphere because motor projections are mostly crossed when they reach the cortex (see Van der Knaap and Van der Ham, 2011 about the inhibitory theory through the corpus callosum to facilitate brain lateralization) it 
remains that during bimanual tasks (e.g. opening a peanut) each hemisphere receives an afferent copy from the opposite hemisphere in order to confront inputs from both sides and perform accurate actions (Brinkman, 1984; Geffen et al. 1994; Andres et al. 1999; Wahl and Ziemann 2008; Liuzzi et al. 2011). In this context, a large connection via the corpus callosum with the opposite cortex was described by Rouiller et al. in 1994 between both SMAs (F3) which are consistent with the functional data (Kermadi et al. 1997; 1998) showing that these structures play a role in the control of bimanual coordinated movements (see Kermadi et al. 2000 for similar conclusions with the cingulate motor cortex, the posterior parietal cortex, F1 and PMd). The anatomical basis of such results have been confirmed in the present work (Figures 4 and 5) but some other additional projections originating from contralateral heterotopic areas state that a broader range of information (most likely inhibitory and excitatory) is transmitted to both PMd and PMv suggesting that PM might be part of a large sensorimotor and multisensory network stretched over the opposite hemisphere. For instance, a recent investigation in humans (Rousseau et al., 2016) concluded that the execution of a vertical hand movement activates a network that includes the contralateral primary motor and somatosensory cortices, PM, SMA, the anterior cerebellum, the cingulate cortex, the prefrontal cortex, the temporal gyrus, the hippocampi (bilateral), and the insula. These anatomical findings help describe the cortical network connected through the corpus callosum which is in position to contribute to sensorimotor and multisensory integration across the two hemispheres.

\section{Multisensory processes}

The present data when brought together in a connectivity matrix (Figure 5) demonstrates that there is a general trend among the two animals characterized by a predominance of homotopic callosal links innervating PMv and PMd. However moderate and sparse projections form a heterotopic network which can be used as a substrate for multisensory integration. Indeed, recent studies support this view and showed various sensory inputs to the two studied premotor areas. Examples include visuomotor behavior (Nelissen et al., 2011; Takahara et al., 2012; Limanowski and Blankenburg, 2016), somatosensory representation (Disbrow et al., 2003; Wardak et al., 2016) and auditory discrimination (Lemus et al., 2009). Premotor fields seem therefore good candidates for passing information from one system to another and hence perform integrative processes. Moreover, we suggest that cortico-cortical callosal pathways could be added to the originally described multisensory integration mechanisms formed by intrahemispheric cortico-cortical loops (Ghazanfar and Schroeder 2006) and thalamocortical 
loops (Cappe et al. 2009). However, if we want to further understand the functional intricacy significance of these cortico-cortical callosal connections it will be necessary to examine neural activity in situ and simultaneously on both sides of the brain.

\section{Applied clinical relevance}

The neurophysiological mechanisms of task related modulation of neural networks have been studied by Merchant et al. (2014). A cognitive task with maximum interactions shows local field potential changes in the corresponding hemisphere as well as in the opposite hemisphere. A longer time lag was observed in the opposite hemisphere. Time lags for negative interactions were longer than for positive interactions in keeping with neuroanatomical measurements (Merchant et al., 2014).

In the context of recovery from a cortical lesion, following a focal lesion in F1, it has been reported in non-human primates that adjacent cortical territories (Nudo and Milliken 1996; Friel and Nudo, 1998) as well as interconnected regions (e.g. premotor cortex, Frost et al. 2003; Dancause et al. 2005) may play a role. Furthermore, when the monkeys were treated with anti-Nogo-A antibody (neutralizing axon growth inhbitors), the callosal connectivity of the premotor cortex was reorganized (Hamadjida et al., 2012). Our anatomical findings support the notion that post-lesional plasticity taking place in one hemisphere might trigger some adaptive changes of the callosal connectivity in case of a unilateral lesion of the premotor area (e.g. apraxia symptoms, Watson and Heilman 1983).

In the context of sensory deprivation in humans suffering from chronic deafness, recent morphometric studies (Penhune et al. 2003; Kara et al. 2006) have reported an increase in the volume of the hand motor area, suggesting cross modal plasticity involving either hemispheres. One could deduce that this compensatory phenomenon of sensory substitution (see for example Rauschecker, 1995; Von Melchner et al., 2000; Finney et al. 2003; Lomber et al. 2010; Barone et al. 2013) points towards the role of the corpus callosum in cortical plasticity. However the speculative nature of these phenomena in humans warrants further experiments to become relevant in clinical practice.

\section{Figures}

Fig 1 A) Upper left: the photomicrograph shows the PMd region of the left hemisphere of Mk-R9 injected with Fluoroemerald (FE). Below that, sections S-15 to S-41 are examples of consecutive coronal slices through the PMd of the same animal displaying a reconstruction of 
the injected site. One can note that the injections covered an area from anteriorly to the genu of the arcuate sulcus (F7) till midway of the spur of the arcuate sulcus (F2). B) The second photomicrograph from the same animal shows the PMv region of the left hemisphere injected with Fast Blue (FB). Examples of corresponding consecutive coronal sections through PMv are displayed as a reconstruction of the injected site. The injections started at the level of the caudal end of the principal sulcus and finished midway over the spur of the arcuate sulcus (therefore in F4 and F5). See list of abbreviations for the lettering.

Fig 2 A) The upper left photomicrograph shows the PMd region of the left hemisphere of MkCI injected with Diamidino Yellow (DY). Below that, sections S-26 to S-49 are examples of consecutive coronal slices through the PMd of the same animal displaying a reconstruction of the injected site. The injections happened from anteriorly to the genu of the arcuate sulcus (F7) till midway of the spur of the arcuate sulcus (F2). B) The second half of the page shows the PMv region of the left hemisphere of the same animal injected with Cholera toxin B subunit (CB). Examples of corresponding consecutive coronal sections through PMv are displayed to present the reconstruction of the injected site. With this marker, the injections were partly in F5 and partly in F4. For the lettering, see list of abbreviations.

Fig 3 Examples of distribution of labeled neurons following injections of FE (green) and FB (blue) in Mk-R9 (A) and DY (orange) and CB (dark blue) in Mk-CI (B), respectively in PMd and PMv. Upper left in both boxes shows sections level $(1,2,3)$ indicated on a lateral view of a schematic macaque brain with aimed injection sites. The grey filled region marks out the block formed before histological work. Upper right in both boxes gives examples of injection site reconstructions (coronal sections taken from Figures 1 and 2). The grey filled regions indicate the injected cortical area according to the parcellation obtained by Saleem and Logothetis (2007). Lower part of both boxes displays coronal sections illustrating retrogradely labeled neurons in parceled cortex (drawing based on the monkey brain atlas) with lettering identifying the areas (see list of abbreviations for the meaning of the acronyms). Below each coronal section the rostrocaudal position of the section is tentatively given relative to the vertical plane passing through the interaural line like in the atlas.

Fig 4 Distribution of callosal neurons in cortical areas connected to PMd (upper graph) and PMv (lower graph) from the opposite hemisphere. The explored cortical areas have been arbitrarily grouped for clarity's sake. The lettering is explained in the abbreviations' list. The 
hatched ( ${ }^{-}$) and black $(\square)$ bars in the histogram correspond to the two different monkeys MkR9 and Mk-CI, respectively.

Fig 5 Individual connectivity matrices for Mk-R9 and Mk-CI. Each row represents one of the 41 separated source areas projecting to one of the two injected target areas under columns PMv or PMd. To better compare labeling distribution between monkeys each target area has been organized into two panels with Mk-R9 on the left and Mk-CI on the right. The labeled neurons are given in terms of logarithmic values ( $\log _{10}$ (ratio)) transformed into positive numbers. The colorbar uses the same scaling for both animals with bright colors representing strong connections (superior or equal to 2.5), medium colors representing moderate connections (between 1.25 and 2.5) and dark colors representing sparse connections (inferior to 1.25$)$.

\section{Glossary}

$\begin{array}{ll}1-2 & : \text { somatosensory areas } 1 \text { and } 2 \\ 3 \mathrm{a} / \mathrm{b} & : \text { somatosensory areas } 3 \mathrm{a} \text { and } 3 \mathrm{~b} \\ 5 & : \text { somatosensory area } 5 \\ 7 \mathrm{~b} & : \text { visual areas } 7 \mathrm{~b} \\ 7 \mathrm{op} & : \text { area } 7 \text { op (parietal operculum) } \\ 8 \mathrm{~A} / \mathrm{Bs} & : \text { frontal eye field areas } \\ 9 & : \text { prefrontal area, dorsal subdivision } \\ 12 \mathrm{o} / \mathrm{l} & : \text { orbitofrontal area } \\ 23 & : \text { area in posterior cingulate cortex } \\ 24 & : \text { area in anterior cingulate cortex } \\ 44,45,46 & : \text { inferior frontal areas } \\ \text { AI } & : \text { auditory area I, core region of the auditory cortex } \\ \text { AIP } & : \text { anterior intraparietal area } \\ \text { AL } & : \text { anterior lateral, belt region of the auditory cortex } \\ \text { CM } & : \text { caudiomedial, belt region of the auditory cortex } \\ \text { G } & : \text { gustatory cortex } \\ \text { Insula } & : \text { agranular, dysgranular and granular insula } \\ \text { IPa } & : \text { area in the superior temporal sulcus } \\ \text { LIP } & : \text { lateral intraparietal area }\end{array}$


: primary motor cortex $(\mathrm{F} 1)$

ML : middle lateral, belt region of the auditory cortex

PGa : area in the superior temporal sulcus

PMd : dorsal premotor cortex (F2 / F7)

$\mathrm{PMv} \quad$ : ventral premotor cortex (F4 / F5)

PrCo : precentral opercular area

Pre-SMA : pre-supplementary motor area (F6)

$\mathrm{R} \quad$ : rostral, core region of the auditory cortex

RM : rostromedial, belt region of the auditory cortex

RTL : lateral rostrotemporal, belt region of the auditory cortex

RTM : medial rostrotemporal, belt region of the auditory cortex

SII : secondary somatosensory area

SMA-proper : supplementary motor area $(\mathrm{F} 3)$

STG : superior temporal gyrus

STGr : rostral superior temporal gyrus

TAa : area in the dorsal bank of the superior temporal sulcus

TE $\quad$ : area in the ventral bank of the superior temporal sulcus

TPO : area in the dorsal bank of the superior temporal sulcus

VIP : ventral intraparietal area

\section{Acknowledgments}

The authors thank Laurent Bossy and Jacques Maillard for their highly valuable daily collaboration for the care of the monkeys in the animal facility, especially before, during and after the various interventions. This work was supported by the Swiss National Science Foundation to Prof. E.M. Rouiller [grant numbers 310000-110005, 31003A-132465 and 310030B-149643]. 
Aboitiz F, Ide A, Olivares R (2003), Corpus callosum morphology in relation to cerebral asymmetries in the postmortem human. In: The parallel brain, chapter 2 (Zaidel E and Iacoboni M, eds), pp 33-46. London: The MIT Press

Andres FG, Mima T, Schulman AE, Dichgans J, Hallett M, Gerloff C (1999), Functional coupling of human cortical sensorimotor areas during bimanual skill acquisition. Brain 122: $855-870$

Barbas H and Pandya DN (1987), Architecture and frontal cortical connections of the premotor cortex (area 6) in the rhesus monkey. J Comp Neurol 256: 211-228

Barone P, Lacassagne L, Kral A (2013), Reorganization of the connectivity of cortical field DZ in congenitally deaf cat. PLoS One 8(4): e60093

Blanchard C, Roll R, Roll JP, Kavounoudias A (2013), Differential contributions of vision, touch and muscle proprioception to the coding of hand movements. PLoS ONE 8(4): e62475

Boussaoud D (1985), Primate premotor cortex: modulation of preparatory neuronal activity by gaze angle. J Neurophysiol 73(2): 886-890

Boussaoud D, Tanné-Gariépy J, Wannier T, Rouiller EM (2005), Callosal connections of dorsal versus ventral premotor areas in the macaque monkey: a multiple retrograde tracing study. BMC Neurosci 6(67). doi: 10.1186/1471-2202-6-67

Brasted PJ and Wise SP (2004), Comparison of learning-related neuronal activity in the dorsal premotor cortex and striatum. Eur J Neurosci 19(3): 721-740

Brinkman C (1984), Supplementary motor area of the monkey's cerebral cortex: short- and long-term deficits after unilateral ablation and the effects of subsequent callosal section. $\mathrm{J}$ Neurosci 4(4): 918-929

Calebrese E, Badea A, Coe CL, Lubach GR, Shi Y, Styner MA, Johnson A (2015), A diffusion tensor MRI atlas of the postmortem rhesus macaque brain. Neuroimage 117: 408416 
Cappe C, Morel A, Rouiller EM (2007), Thalamocortical and the dual pattern of corticothalamic projections of the posterior parietal cortex in macaque monkeys. Neuroscience 146:1371-87

Cappe C, Morel A, Barone P, Rouiller EM (2009), The thalamocortical projection systems in primate: an anatomical support for multisensory and sensorimotor interplay. Cereb Cortex 19(9):2025-37

Cisek P and Kalaska JF (2005), Neural correlates of reaching decisions in dorsal premotor cortex: specification of multiple direction choices and final selection of action. Neuron 45(5): 801-814

Clarke S and Miklossy J (1990), Occipital cortex in man: Organization of callosal connections, related myelo- and cytoarchitecture, and putative boundaries of functional visual areas. J Comp Neurol 298:188-214

Clarke S (1994), Association and intrinsic connections of human extrastriate visual cortex. P R Soc London B 257: 87-89

Clarke S (2003), Complexity of human interhemispheric connections. In: The parallel brain, commentary 2.1 (Zaidel E and Iacobini M, eds), pp 47-49. London, the MIT Press

Coudè G, Ferrari PF, Rodà F, Maranesi M, Borelli E, Veroni V, Monti F, Rozzi S, Fogassi L (2011), Neurons controlling voluntary vocalization in the macaque ventral premotor cortex. PLoS One 6(11), e26822

Dancause N, Barbay S, Frost SB, Plautz EJ, Chen DF, Zoubina EV, Stowe AM, Nudo RJ (2005), Extensive cortical rewiring after brain injury. J Neurosci 25: 10167-10179

Di Virgilio G and Clarke S (1997), Direct interhemispheric visual input to human speech areas. Hum Brain Mapp 5: 347-354 
Disbrow E, Litinas E, Recanzone GH, Padberg J, Krubitzer L (2003), Cortical connections of the second somatosensory area and the parietal ventral area in macaque monkeys. J Comp Neurol 462:382-399

Driver J and Noesselt T (2008), Multisensory interplay reveals crossmodal influences on "sensory specific" brain regions, neural responses, and judgments. Neuron 57: 11-23

Dubach MF and Bowden DM (2009), BrainInfo online 3D macaque brain atlas: a database in the shape of a brain. Soc Neur Ann Meet, Chicago, IL Abstract No 199.5

Fabri M, Polonra G, Mascioli G, Salvolini U, Manzoni T (2011), Topographical organization of human corpus callosum: an fMRI mapping study. Br Res 1370: 99-111.

Finney EM, Clementz BA, Hickok G, Dobkins KR (2003), Visual stimuli activate auditory cortex in deaf subjects: evidence from MEG. Neuroreport 14: 1425-1426

Fogassi L, Gallese V, Fadiga L, Luppino G, Matelli M, Rizzolatti G (1996), Coding of peripersonal space in inferior premotor cortex (area F4). J Neurophysiol 76(1): 141-157

Friel KM and Nudo RJ (1998), Recovery of motor function after cortical injury in primates: compensatory movement patterns used during rehabilitative training. Somatosens Mot Res 15(3): 173-189

Frost SB, Barbay S, Friel KM, Plautz EJ, Nudo RJ (2003), Reorganization of remote cortical regions after ischemic brain injury: a potential substrate for stroke recovery. J Neurophysiol 89: $3205-3214$

Geffen GM, Jones DL, Geffen LB (1994), Interhemispheric control of manual motor activity. Behav Brain Res 64(1-2): 131-140

Ghazanfar AA and Schroeder CE (2006), Is neocortex essentially multisensory? Trends Cogn Sci 10(6): 278-285 
Giard MH and Peronnet F (1999), Auditory-visual integration during multimodal object recognition in humans: a behavioral and electrophysiological study. J Cognitive Neurosci 11: $473-490$

Ghosh S, Brinkmann C, Porter R (1987), A quantitative study of the distribution of neurons projecting to the precentral motor cortex in the monkey (M. fascicularis). J Comp Neurol 259: $424-444$

Ghosh S and Gattera R (1995), A comparison of the ipsilateral cortical projections to the dorsal and ventral subdivisions of the macaque premotor cortex. Somatosens Mot Res 12(34): $359-378$

Godschalk M, Lemon RN, Kuypers HG, Ronday HK (1984), Cortical afferents and efferents of monkey postarcuate area: an anatomical and electrophysiological study. Exp Brain Res 56: $410-424$

Grafton ST and Hamilton AF (2007), Evidence for a distributed hierarchy of action representation in the brain. Hum Movement Sci 26: 590-616

Graziano MSA, Yap GS, Gross CG (1994), Coding of visual space by premotor neurons. Science 266(5187): 1054-1057

Graziano MSA, Hu X, Gross CG (1997), Visuo-spatial properties of ventral premotor cortex. J Neurophysiol 77: 2268-2292

Graziano MSA, Reiss LAJ, Gross CG (1999), A neuronal representation of the location of nearby sounds. Nature 397(4): 428-430

Hamadjida A, Wyss AF, Mir A, Schwab ME, Belhaj-Saif A, Rouiller EM (2012), Influence of anti-Nogo-A antibody treatment on the reorganization of callosal connectivity of the premotor cortical areas following unilateral lesion of primary motor cortex (M1) in adult macaque monkeys. Exp Brain Res 223(3): 321-340 
Hofer S, Frahm J (2006), Topography of the human corpus callosum revisited -

Comprehensive fiber tractography using diffusion tensor magnetic resonance imaging.

Neuroimage 32: 989-994

Innocenti GM (1986), General organization of callosal connections in the cerebral cortex. In:

Cerebral Cortex, vol. 5 (Jones EG and Peters A, eds), pp 291-354. Plenum: New York

Innocenti GM (1994), Some new trends in the study of the corpus callosum. Behav Brain Res 64: $1-8$

Innocenti GM (1995), Cellular aspects of callosal connections and their development. Neuropsychologia 33: 961-988

Jenny AB (1979), Commissural projections of the cortical hand motor area in monkeys. J Comp Neurol 188: 137-146

Kara A, Hakan Ozturk A, Kurtoglu Z, Umit Talas D, Aktekin M, Saygili M, Kanik A (2006), Morphometric comparison of the human corpus callosum in deaf and hearing subjects: an MRI study. J Neuroradiology 33: 158-163

Keele SW, Cohen A, Ivry R (1990), Motor programs: concepts and issues. Chapter 3. In: Jeannerod M (ed) Attention and performance 13: Motor representation and control. Hillsdale, NJ, USA: Lawrence Erlbaum Associates, Inc.

Kermadi I, Liu Y, Tempini A, Rouiller EM (1997), Effects of reversible inactivation of the supplementary motor area (SMA) on unimanual grasp and bimanual pull and grasp performance in monkeys. Somatosens Mot Res 14: 268-280

Kermadi I, Liu Y, Tempini A, Calciati E, Rouiller EM (1998), Neuronal activity in the primate supplementary motor area and the primary motor cortex in relation to spatio-temporal bimanual coordination. Somatosens Mot Res 15(4): 287-308 
Kermadi I, Liu Y, Rouiller EM (2000), Do bimanual motor actions involve the dorsal premotor (PMd), cingulate (CMA) and posterior parietal (PPC) cortices? Comparison with primary and supplementary motor cortical areas. Somatosens Mot Res 17(3): 255-271

Kohler E, Keysers C, Umiltà MA, Fogassi L, Gallese V, Rizzolatti G (2002), Hearing sounds, understanding actions: action representation in mirror neurons. Science 2(297): 846-848

Kurata K (1991), Corticocortical inputs to the dorsal and ventral aspects of the premotor cortex of macaque monkeys. Neurosci Res 12: 263-280

Lanz F, Moret V, Rouiller EM, Loquet G (2013), Multisensory integration in non-human primates during a sensory-motor task. Frontier Hum Neurosci 7(799): 1-15

Lemus L, Hernandez A, Romo R (2009), Neural encoding of auditory discrimination in ventral premotor cortex. Proc Natl Acad Sci USA 106:14640-14645

Limanowski J, Blankenburg F (2016), Integration of Visual and Proprioceptive Limb Position Information in Human Posterior Parietal, Premotor, and Extrastriate Cortex. J Neurosci $36: 2582-2589$

Liu J, Morel A, Wannier T, Rouiller EM (2002), Origins of callosal projections to the supplementary motor area (SMA): A direct comparison between pre-SMA and SMA-proper in macaque monkeys. J Comp Neurol 443:71-85

Liuzzi G, Hörniß M, Zimermann, Gerloff C, Hummel FC (2011), Coordination of uncoupled bimanual movements by strictly timed interhemispheric connectivity. J Neurosci 31(25):

9111-9117

Lomber SG, Meredith MA, Kral A (2010), Cross-modal plasticity in specific auditory cortices underlies visual compensations in the deaf. Nat Neurosci 13: 1421-7

Marconi B, Genovesio A, Giannetti S, Molinari M, Caminiti R (2003), Callosal connections of dorso-lateral premotor cortex. Eur J Neurosci 18: 775-788 
Markov NT, Ercsey-Ravasz MM, Ribeiro Gomes AR, Lamy C, Magrou L, Vezoli J, Misery

P, Falchier A, Quilodran R, Gariel MA, Sallet J, Gamanut R, Huissoud C, Clavagnier S, Giroud P, Sappey-Marinier D, Barone P, Dehay C, Toroczkai Z, Knoblauch K, Van Essen DC, Kennedy H (2014), A weighted and directed interareal connectivity matrix for macaque cerebral cortex. Cereb Cortex 24: 17-36

Matelli M, Camarda R, Glickstein M, Rizzolatti G (1984), Interconnections within the postarcuate cortex (area 6) of the macaque monkey. Brain Res 310: 388-392

Matelli M, Camarda R, Glickstein M, Rizzolatti G (1986), Afferent and efferent projections of the inferior area 6 in the macaque monkey. J Comp Neurol 251: 281-298

Merchant H, Crowe DA, Fortes AF, Georgopoulos AP (2014), Cognitive modulation of local and callosal neural interactions in decision making. Front Neurosci 245: 1-10

Morecraft RJ, Stilwell-Morecraft KS, Cipolloni PB, Ge J, McNeal DW, Pandya DN (2012), Cytoarchitecture and cortical connections of the anterior cingulate and adjacent somatomotor fields in the rhesus monkey. Brain Res Bull 87(4-5): 457-497

Morel A, Liu J, Wannier T, Jeanmonod D, Rouiller EM (2005), Divergence and convergence of thalamocortical projections to premotor and supplementary motor cortex: a multiple tracing study in macaque monkey. Eur J Neurosci 21:1007-29

Nelissen K, Borra E, Gerbella M, Rozzi S, Luppino G, Vanduffel W, Rizzolatti G, Orban GA (2011), Action observation circuits in the macaque monkey cortex. J Neurosci 31:3743-3756

Nudo RJ and Milliken GW (1996), Reorganization of movement representations in primary motor cortex following focal ischemic infarcts in adult squirrel monkeys. J Neurophysiol 75: 2144-2149

Paxinos G, Huang XF, Toga AW (2000), The rhesus monkey brain in stereotaxic coordinates. Academic Press, San Diego 
Pandya DN and Vignolo LA (1971), Intra- and interhemispheric projections of the precentral, premotor and arcuate areas in the rhesus monkey. Brain Res 26(2): 217-233

Penhune VB, Cismaru R, Dorsaint-Pierre R, Petitto LA, Zatorrec RJ (2003), The morphometry of auditory cortex in the congenitally deaf measured using MRI. Neuroimage $1215-1225$

Phillips KA and Hopkins WD (2012), Topography of the Chimpanzee corpus callosum. PLoS ONE, 7(2): e31941. doi:10.1371/journal.pone.0031941

Rauschecker JP (1995), Compensatory plasticity and sensory substitution in the cerebral cortex. Trends Neurosci 18: 36-43

Rizzolatti G, Luppino G, Matelli M (1998), The organization of the cortical motor system: new concepts. Electroen Clin Neuro 106: 283-296

Rizzolatti G and Luppino G (2001), The cortical motor system. Neuron 31(27): 889-901

Rohlfing T, Kroenke CD, Sullivan EV, Dubach MF, Bowden DM, Grant KA, Pfefferbaum A (2012), The INIA 19 template and neuromaps atlas for primate brain image parcellation and spatial normalization. Front Neuroinf 6:27

Rouiller EM, Babalian A, Kazennikov O, Moret V, Yu X-H, Wiesendanger M (1994), Transcallosal connections of the distal forelimb representations of the primary and supplementary motor cortical areas in macaque monkeys. Exp Brain Res 102: 227-243

Rousseau C, Fautrelle L, Papaxanthis C, Fadiga L, Pozzo T, White O (2016), Directiondependent activation of the insular cortex during vertical and horizontal hand movements. Neurosci 325: 10-19

Saleem KS and Logothetis NK (2007), A combined MRI and histology atlas of the rhesus monkey brain in stereotaxic coordinates. Academic Press, Elsevier

Stein BE and Meredith MA (1993), The merging of the senses. Cambridge, MA: MIT Press 
Takahara D, Inoue KI, Hirata Y, Miyachi S, Nambu A, Takada M, Hoshi E (2012), Multisynaptic projections from the ventrolateral prefrontal cortex to the dorsal premotor cortex in macaques - anatomical substrate for conditional visuomotor behavior. Eur $\mathbf{J}$ Neurosci 36:3365-3375

Tanné-Gariépy J, Rouiller EM, Boussaoud D (2002), Parietal inputs to dorsal versus ventral premotor areas in the macaque monkey: evidence for largely segregated visuomotor pathways. Exp Brain Res 145: 91-103

Van Essen DC (2002), Surface-based atlases of cerebellar cortex in the human, macaque and mouse. Ann N Y Acad Sci 978: 468-479

Van der Knaap LJ and Van der Ham IJ (2011), How does the corpus callosum mediate interhemispheric transfer? A review. Behav Brain Res 223(1): 211-221

Von Melchner L, Pallas SL, Sur M (2000), Visual behaviour mediated by retinal projections directed to the auditory pathway. Nature 404(6780): 871-876

Wahl M and Ziemann U (2008), The human motor corpus callosum. Rev Neurosci 19(6): $451-466$

Wardak C, Guipponi O, Pinede S, Ben HS (2016), Tactile representation of the head and shoulders assessed by fMRI in the nonhuman primate. J Neurophysiol 115:80-91

Watson RT and Heilman KM (1983), Callosal apraxia. Brain, 106(Pt 2):391-403

Weinrich M and Wise SP (1982), The premotor cortex of the monkey. J Neurosci 2: 13291345

Weinrich M, Wise SP, Mauritz KH (1984), A neurophysiological study of the premotor cortex in the rhesus monkey. Brain 107: 385-414 
Wise SP, Boussaoud D, Johnson PB, Caminiti R (1997), Premotor and parietal cortex:

corticocortical connectivity and combinatorial computations. Annu Rev Neurosci 20: 25-42 

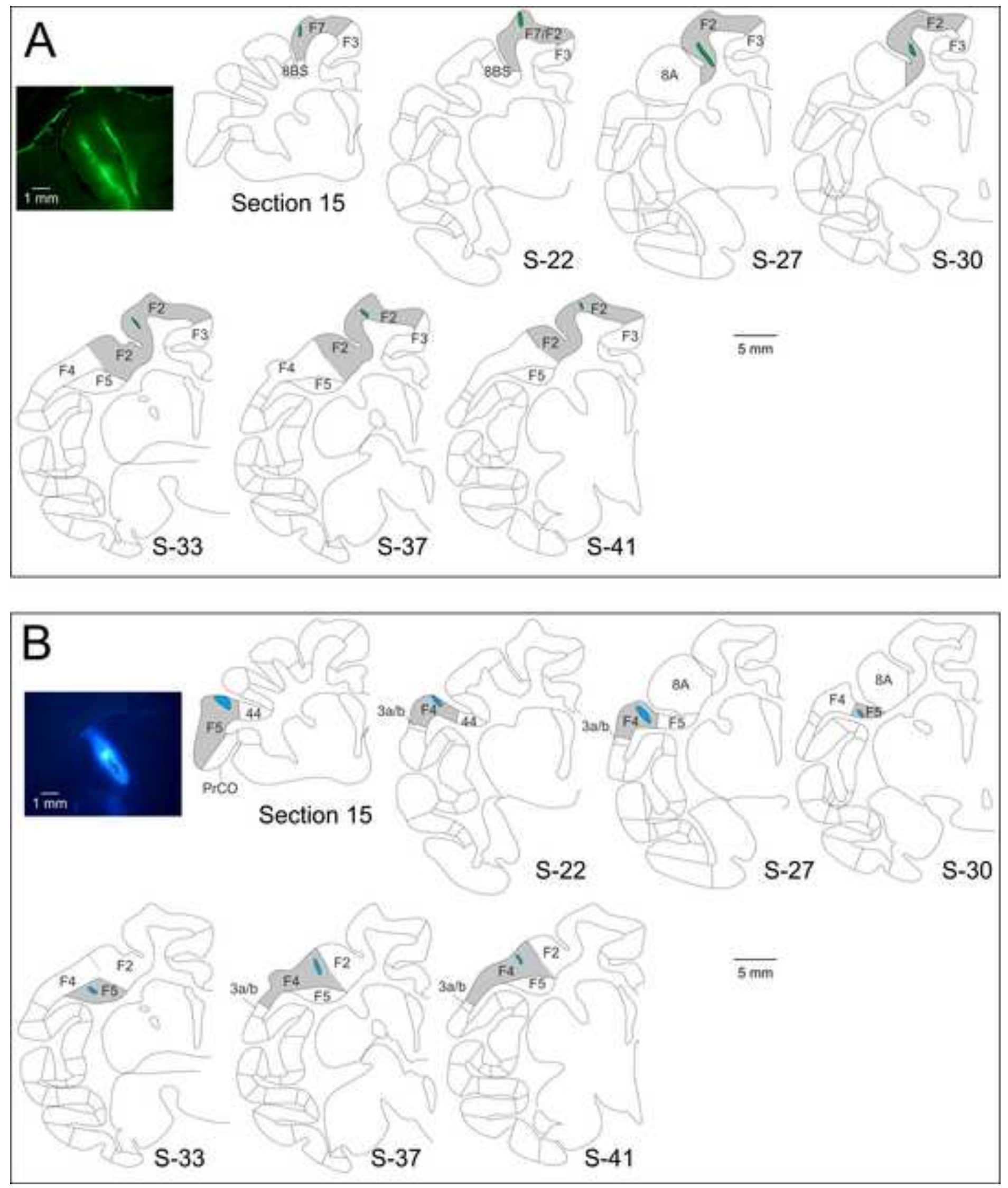


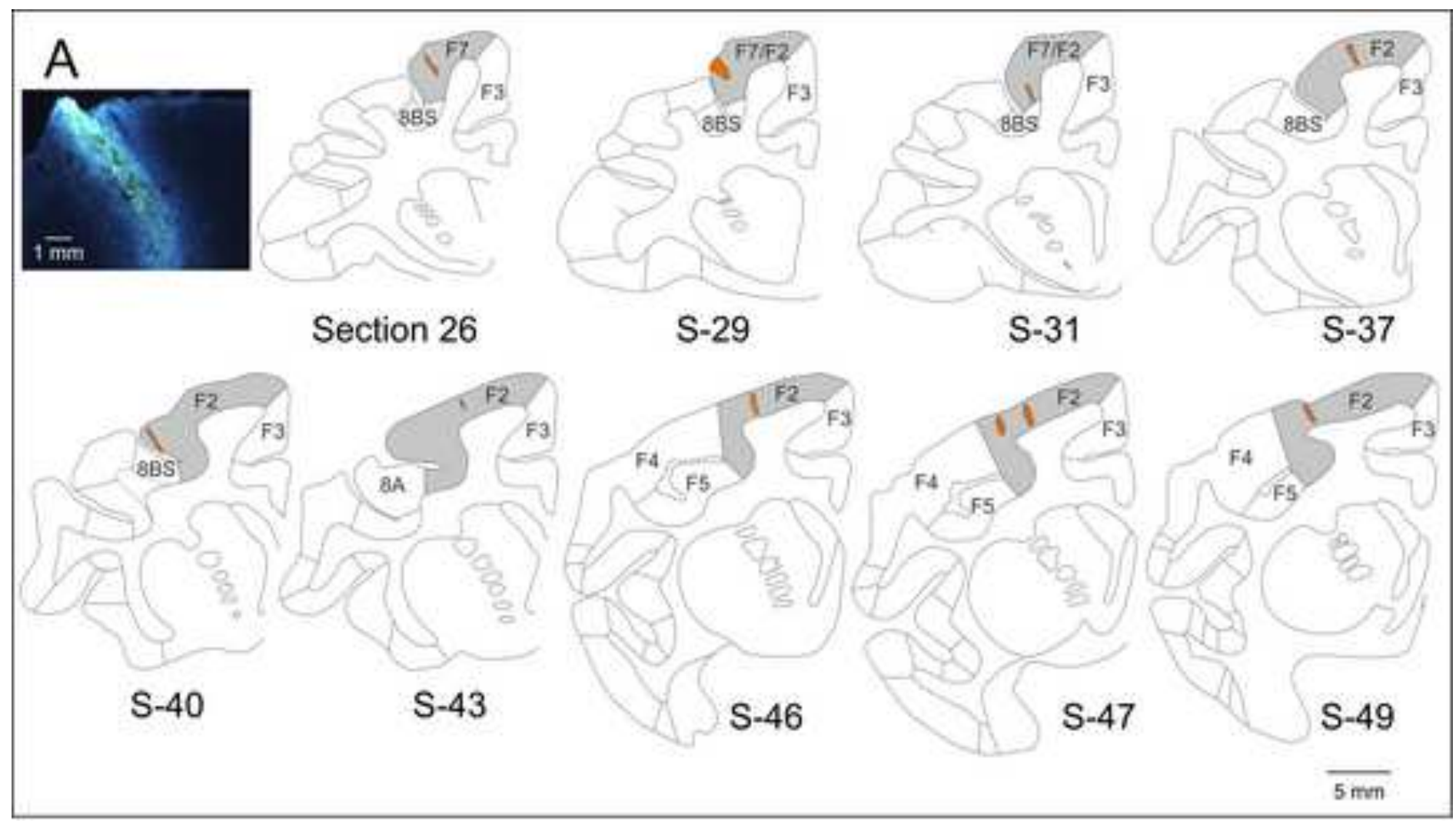

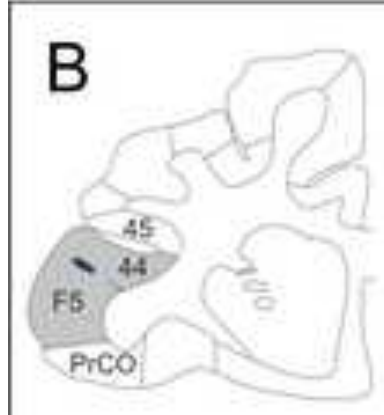

Section 29

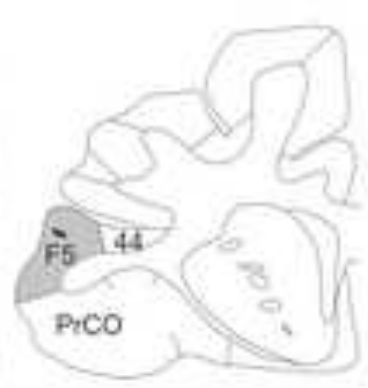

S-31

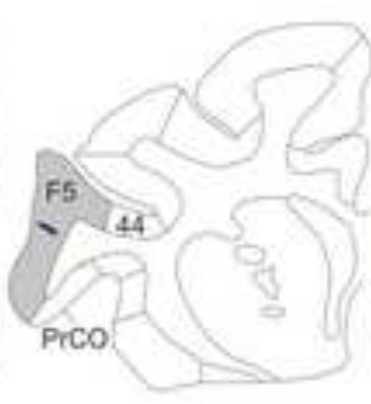

S-37
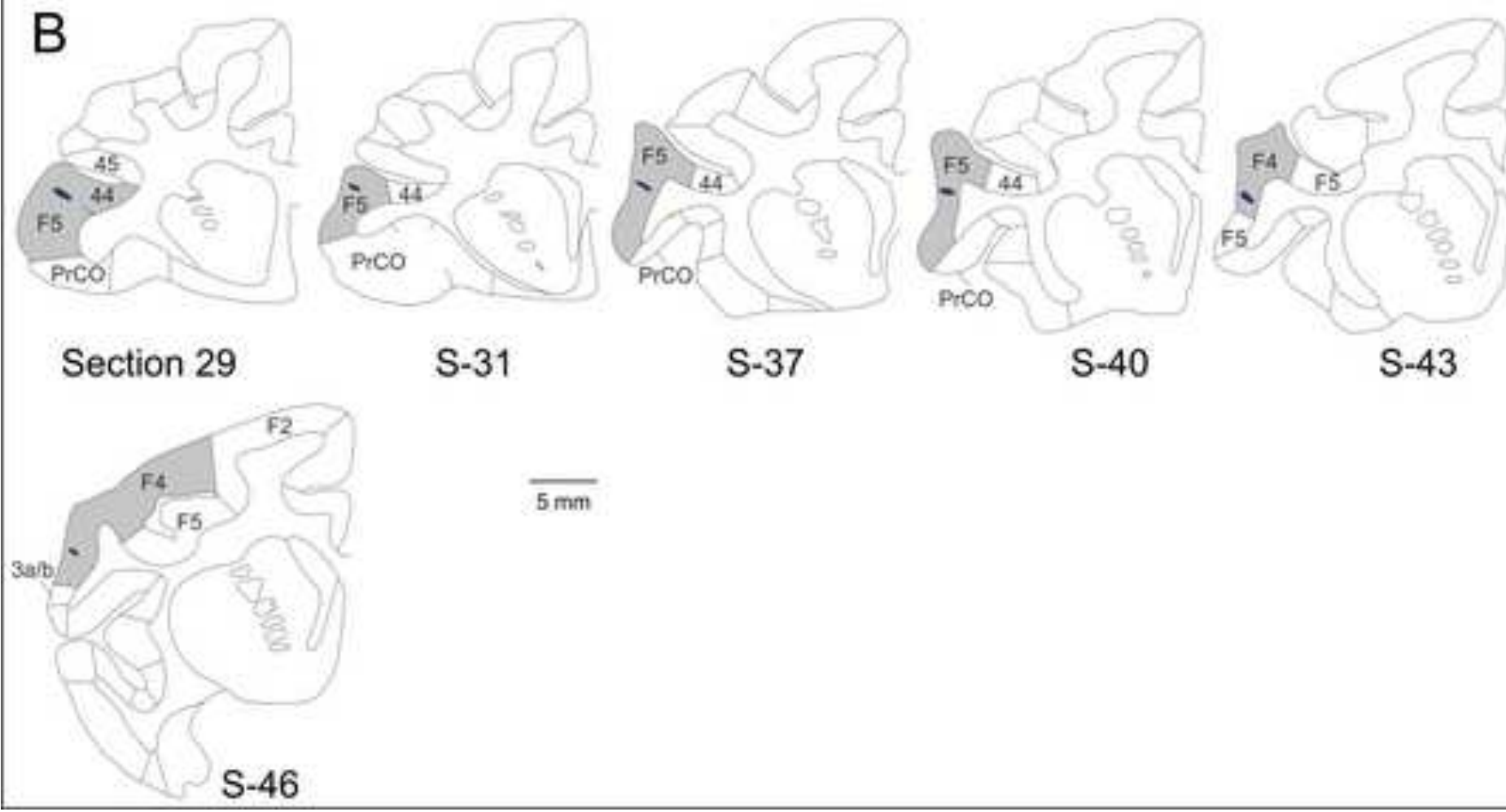

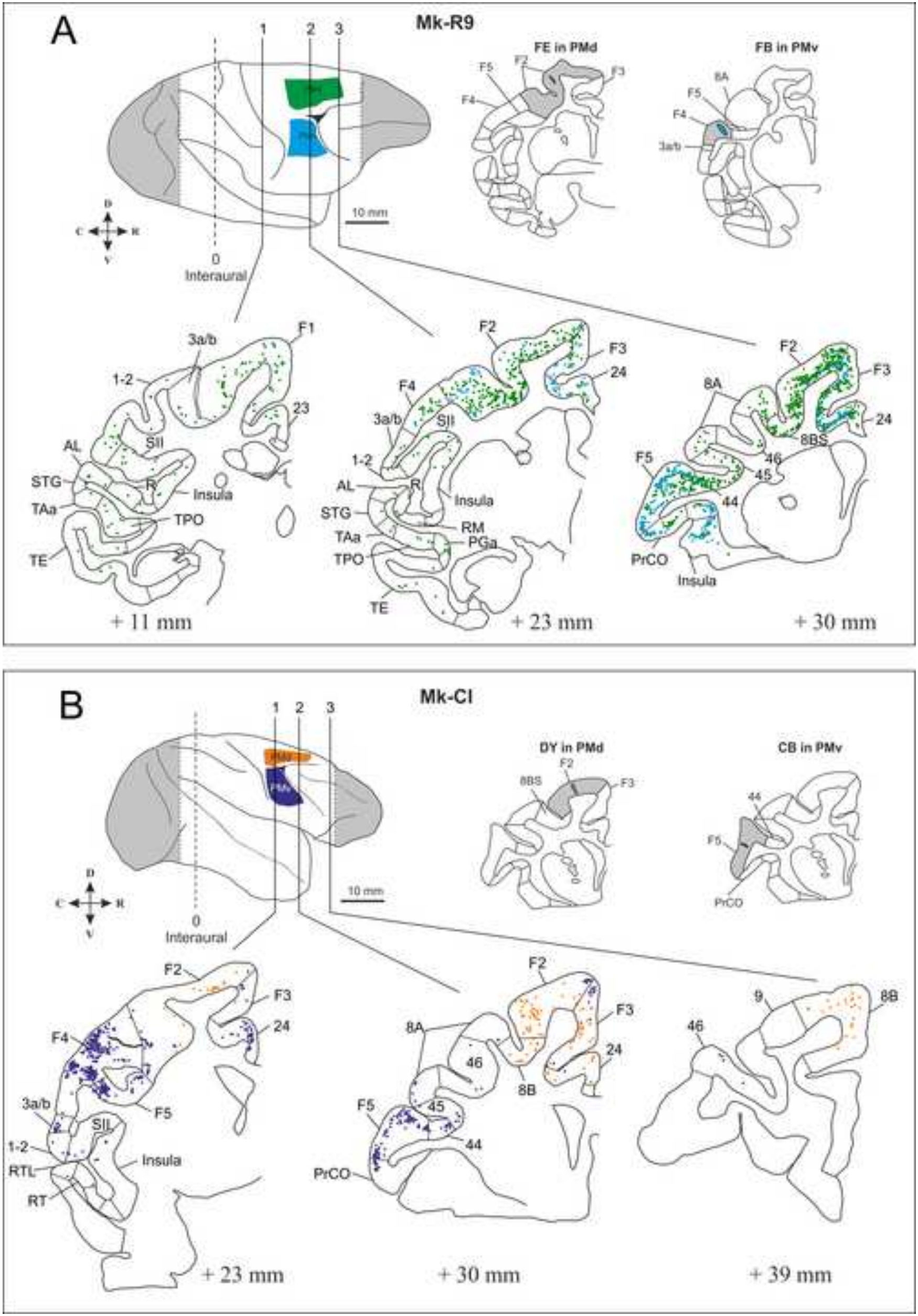

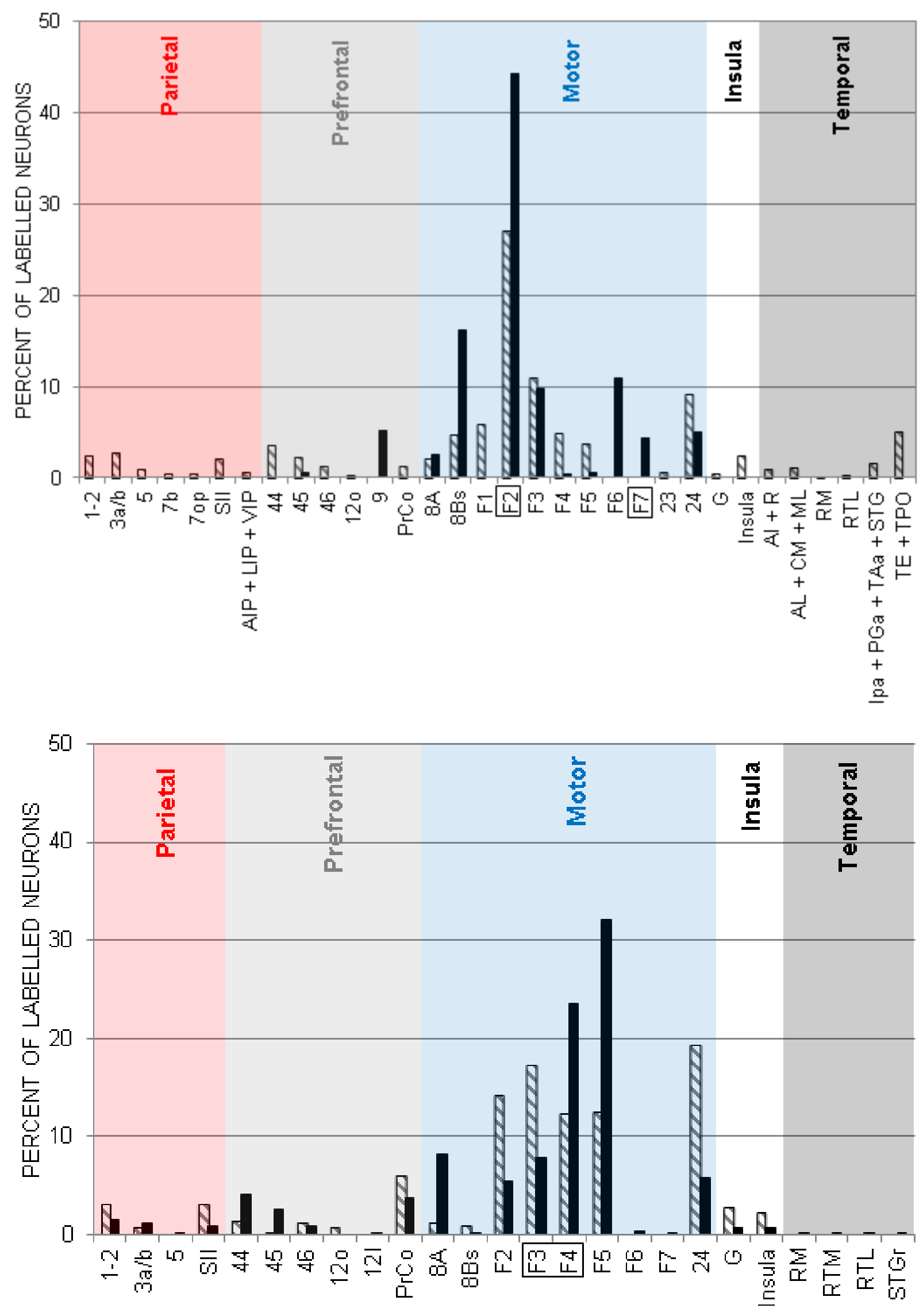
TARGET AREAS

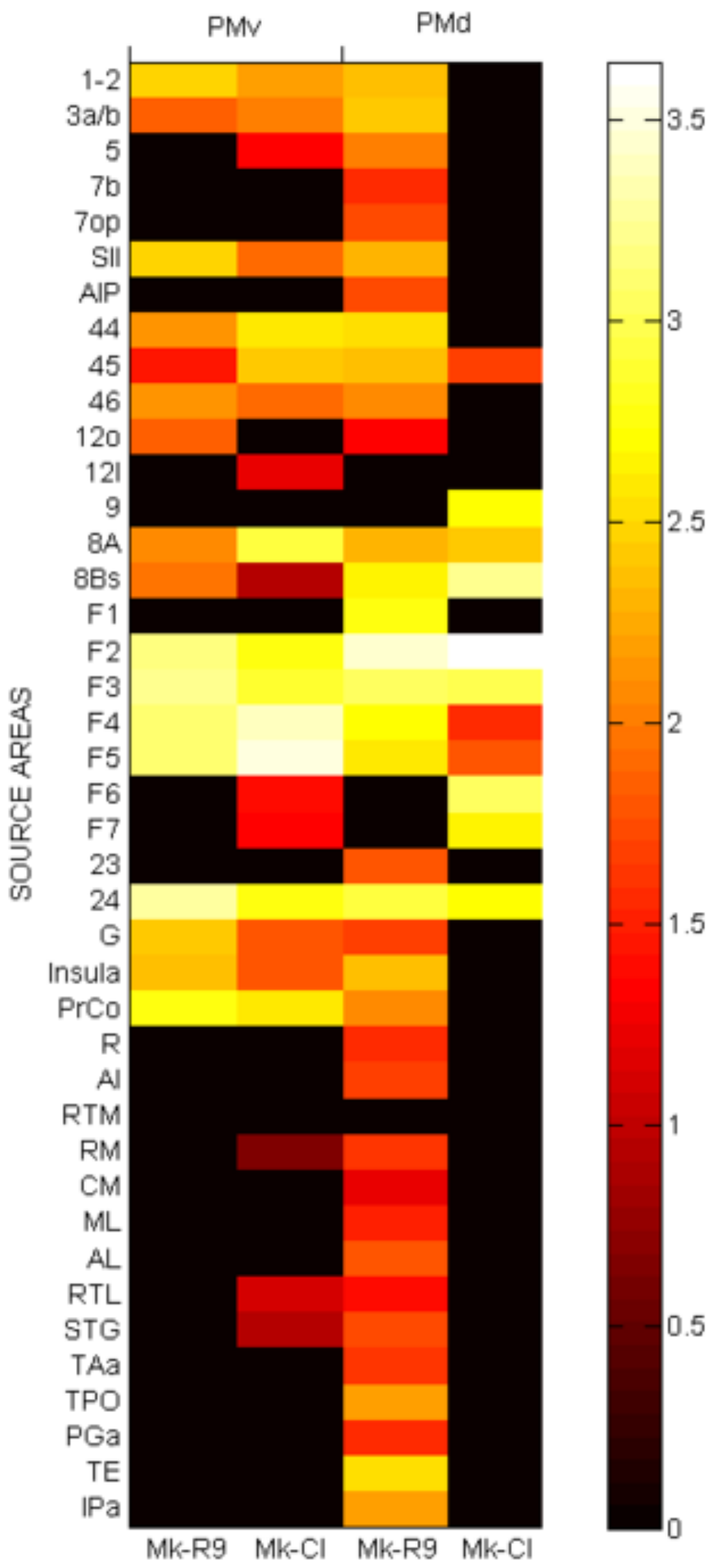

